\title{
ON THE LOCATION OF THE $\gamma$-RAY OUTBURST EMISSION IN THE BL LACERTAE OBJECT AO $0235+164$ THROUGH OBSERVATIONS ACROSS THE ELECTROMAGNETIC SPECTRUM
}

\author{
Iván Agudo ${ }^{1,2}$, Alan P. Marscher ${ }^{2}$, Svetlana G. Jorstad ${ }^{2,3}$, VAleri M. Larionov $^{3,4}$, José L. Gómez $^{1}$, \\ Anne LähteenmäKi ${ }^{5}$, Paul S. Smith ${ }^{6}$, Kari Nilsson ${ }^{7}$, Anthony C. S. Readhead $^{8}$, Margo F. Aller $^{9}$, Jochen Heidt $^{10}$, \\ Mark Gurwell $^{11}$, Clemens Thum $^{12}$, Ann E. Wehrle ${ }^{13}$, Maria G. Nikolashvili ${ }^{14}$, Hugh D. Aller ${ }^{9}$, Erika Benítez ${ }^{15}$, \\ Dmitriy A. Blinov ${ }^{3,4}$, Vladimir A. Hagen-Thorn ${ }^{3,4}$, David Hiriart $^{16}$, Buell T. Jannuzi ${ }^{17}$, Manasvita Joshi ${ }^{2}$, \\ Givi N. Kimeridze ${ }^{14}$, Omar M. Kurtanidze ${ }^{14}$, Sofia O. Kurtanidze ${ }^{14}$, Elina Lindfors ${ }^{18}$, Sol N. Molina ${ }^{1}$, \\ Daria A. Morozova ${ }^{3}$, Elina Nieppola ${ }^{5,7}$, Alice R. Olmstead ${ }^{2}$, Riho Reinthal $^{18}$, Mar Roca-Sogorb $^{1}$, Gary D. Schmidt ${ }^{19}$, \\ Lorand A. Sigua ${ }^{14}$, Aimo Sillanpä ${ }^{18}{ }^{18}$ Leo Takalo $^{18}$, Brian Taylor ${ }^{2,20}$, Merja Tornikoski ${ }^{5}$ Ivan S. Troitsky $^{3}$, \\ Alma C. ZOOK ${ }^{21}$, AND Helmut Wiesemeyer ${ }^{22}$ \\ ${ }^{1}$ Instituto de Astrofísica de Andalucía, CSIC, Apartado 3004, 18080 Granada, Spain; iagudo@iaa.es \\ ${ }^{2}$ Institute for Astrophysical Research, Boston University, 725 Commonwealth Avenue, Boston, MA 02215, USA \\ ${ }^{3}$ Astronomical Institute, St. Petersburg State University, Universitetskij Pr. 28, Petrodvorets, 198504 St. Petersburg, Russia \\ ${ }^{4}$ Isaac Newton Institute of Chile, St. Petersburg Branch, St. Petersburg, Russia \\ ${ }^{5}$ Aalto University Metsähovi Radio Observatory, Metsähovintie 114, FIN-02540 Kylmälä, Finland \\ ${ }^{6}$ Steward Observatory, University of Arizona, Tucson, AZ 85721-0065, USA \\ ${ }^{7}$ Finnish Centre for Astronomy with ESO (FINCA), University of Turku, Väisäläntie 20, FIN-21500 Piikkiö, Finland \\ ${ }^{8}$ Cahill Center for Astronomy and Astrophysics, California Institute of Technology, Mail Code 222, Pasadena, CA 91125 , USA \\ ${ }^{9}$ Department of Astronomy, University of Michigan, 817 Dennison Building, Ann Arbor, MI 48 109, USA \\ ${ }^{10}$ ZAH, Landessternwarte Heidelberg, Königstuhl, 69117 Heidelberg, Germany \\ ${ }^{11}$ Harvard-Smithsonian Center for Astrophysics, 60 Garden St., Cambridge, MA 02138, USA \\ ${ }^{12}$ Institut de Radio Astronomie Millimétrique, 300 Rue de la Piscine, 38406 St. Martin d'Hères, France \\ ${ }^{13}$ Space Science Institute, Boulder, CO 80301, USA \\ ${ }^{14}$ Abastumani Observatory, Mt. Kanobili, 0301 Abastumani, Georgia \\ ${ }^{15}$ Instituto de Astronomía, Universidad Nacional Autónoma de México, 04510 México D. F., Mexico \\ ${ }^{16}$ Instituto de Astronomía, Universidad Nacional Autónoma de México, 2280 Ensenada, Mexico \\ ${ }^{17}$ National Optical Astronomy Observatory, KPNO, Tucson, AZ 85726, USA \\ ${ }^{18}$ Tuorla Observatory, University of Turku, Väisäläntie 20, FIN-21500 Piikkiö, Finland \\ ${ }^{19}$ National Science Foundation, 4201 Wilson Blvd., Arlington, VA 22230, USA \\ ${ }^{20}$ Lowell Observatory, Flagstaff, AZ 86001, USA \\ ${ }^{21}$ Department of Physics and Astronomy, Pomona College, Claremont, CA 91711, USA \\ 22 Instituto de Radio Astronomía Milimétrica, Avenida Divina Pastora, 7, Local 20, E-18012 Granada, Spain \\ Received 2011 April 27; accepted 2011 May 18; published 2011 June 6
}

\begin{abstract}
We present observations of a major outburst at centimeter, millimeter, optical, X-ray, and $\gamma$-ray wavelengths of the BL Lacertae object AO $0235+164$. We analyze the timing of multi-waveband variations in the flux and linear polarization, as well as changes in Very Long Baseline Array images at $\lambda=7 \mathrm{~mm}$ with $\sim 0.15$ milliarcsec resolution. The association of the events at different wavebands is confirmed at high statistical significance by probability arguments and Monte Carlo simulations. A series of sharp peaks in optical linear polarization, as well as a pronounced maximum in the $7 \mathrm{~mm}$ polarization of a superluminal jet knot, indicate rapid fluctuations in the degree of ordering of the magnetic field. These results lead us to conclude that the outburst occurred in the jet both in the quasi-stationary "core" and in the superluminal knot, both parsecs downstream of the supermassive black hole. We interpret the outburst as a consequence of the propagation of a disturbance, elongated along the line of sight by light-travel time delays, that passes through a standing recollimation shock in the core and propagates down the jet to create the superluminal knot. The multi-wavelength light curves vary together on long timescales (months/years), but the correspondence is poorer on shorter timescales. This, as well as the variability of the polarization and the dual location of the outburst, agrees with the expectations of a multi-zone emission model in which turbulence plays a major role in modulating the synchrotron and inverse Compton fluxes.
\end{abstract}

Key words: BL Lacertae objects: individual (AO 0235+164) - galaxies: active - galaxies: jets - gamma rays: general - polarization - radio continuum: galaxies

\section{INTRODUCTION}

Our understanding of the processes leading to the generation of $\gamma$-ray emission from blazars, the most extreme active galactic nuclei, depends on where those $\gamma$-rays originate. This is currently the subject of considerable debate (e.g., Marscher \& Jorstad 2010; Tavecchio et al. 2010; Agudo et al. 2011). Two main locations of the site of $\gamma$-ray emission in blazars have been proposed. The first is close $(\lesssim 0.1-1$ pc) to the supermas- sive black hole $(\mathrm{BH})$, which can readily explain short timescales of variability of a few hours reported in some $\gamma$-ray observations of blazars (Ackermann et al. 2010; Foschini et al. 2010, 2011; Tavecchio et al. 2010). Although short timescales of variability limit the size of the emission region and do not necessarily imply short distances to the $\mathrm{BH}$, this scenario has the advantage that optical-UV photons from the broad emission-line region are available for scattering to $\gamma$-ray energies by highly relativistic electrons in the jet. 
However, locating the $\gamma$-ray emission region so close to the $\mathrm{BH}$ contradicts the increasingly large number of time coincidences of radio-millimeter and $\gamma$-ray events (Jorstad et al. 2010; Marscher et al. 2010; Agudo et al. 2011). This problem is overcome if the $\gamma$-rays are emitted from a region much farther $(\gg 1 \mathrm{pc})$ from the $\mathrm{BH}$, beyond the "core" where the jet starts to be visible at millimeter wavelengths with very long baseline interferometry (VLBI). Supporting this second scenario, Agudo et al. (2011) unambiguously locate the region of $\gamma$-ray flares $>14$ pc from the BH in the jet of OJ287 through correlation of millimeter wave with $\gamma$-ray light curves and direct ultrahighresolution $7 \mathrm{~mm}$ imaging with the Very Long Baseline Array (VLBA). Similar results are obtained by Marscher et al. (2010) and Jorstad et al. (2010) for PKS 1510-089 and 3C 454.3, respectively. Marscher \& Jorstad (2010) have recently proposed a model that reconciles both $\gamma$-ray flare emitting regions located at $\gg 1 \mathrm{pc}$ from the $\mathrm{BH}$ and intraday $\gamma$-ray variability through a model involving turbulent plasma flowing through standing shocks in the core with a volume filling factor that decreases with energy and therefore frequency.

This Letter investigates the location and properties of a radio to $\gamma$-ray outburst in the BL Lacertae object AO $0235+164$ $(0235+164$ hereafter, $z=0.94)$. Historically, the blazar has exhibited extreme variability (by over an order of magnitude on timescales $<1$ year) across all spectral ranges, including X-ray (e.g., Raiteri et al. 2009) and $\gamma$-ray (e.g., Abdo et al. 2010a). The most prominent radio outbursts are accompanied by optical counterparts (Ledden et al. 1976; Balonek \& Dent 1980; Raiteri et al. 2008), although no correlation with variability at other wavebands has been reported previously.

On submilliarcsecond scales, $0235+164$ is extremely compact $(\lesssim 0.5$ mas $)$ at millimeter wavelengths (e.g., Piner et al. 2006) and shows extreme superluminal apparent speeds $\left(\beta_{\mathrm{app}}^{\max }=46.5 \pm 8.0 \mathrm{c}\right.$; Jorstad et al. 2001). ${ }^{23}$ This speed, and the variability Doppler factor $\delta_{\mathrm{var}}=24.0$ of $0235+164$ (Hovatta et al. 2009), sets a maximum jet viewing angle $\theta=\arctan \left[2 \beta_{\mathrm{app}}^{\max } /\left(\beta_{\mathrm{app}}^{\max 2}+\delta_{\mathrm{var}}^{2}-1\right)\right] \lesssim 2.4$. Although the large $\beta_{\text {app }}^{\max }$ value might lead to underestimation of $\theta$ for non-cylindric jets (Gopal-Krishna et al. 2006), the unusual compactness of $0235+164$ and its extreme flaring activity are consistent with low $\theta$ values as estimated above (see also Hovatta et al. 2009). Superluminal knots have been observed at position angles ranging from $5^{\circ}$ to $-55^{\circ}$ (Jorstad et al. 2001; Piner et al. 2006), consistent with a broad $\left(\alpha_{\text {app }} \approx 60^{\circ}\right)$ projected jet opening angle. Both $\theta$ and $\alpha_{\text {app }}$ constrain the maximum intrinsic jet half-opening-angle $\alpha_{\text {int }} / 2=\left(\alpha_{\text {app }} \sin \theta\right) / 2 \lesssim 1.25$.

\section{OBSERVATIONS}

Our photo-polarimetric monitoring observations of $0235+164$ (Figures 1-3) include (1) $7 \mathrm{~mm}$ images with the VLBA from the Boston University monthly blazar-monitoring program, ${ }^{24}$ (2) $3 \mathrm{~mm}$ observations with the Instituto de Radioastronomía Milimétrica (IRAM) $30 \mathrm{~m}$ Telescope, and (3) optical measurements from the following telescopes: Calar Alto (2.2 $\mathrm{m}$ Telescope, observations under the MAPCAT ${ }^{25}$ program), Steward Observatory (2.3 and $1.54 \mathrm{~m}$ Telescopes ${ }^{26}$ ),

\footnotetext{
23 We adopt the standard $\Lambda$ CDM cosmology, with $H_{0}=71 \mathrm{~km} \mathrm{~s}^{-1} \mathrm{Mpc}^{-1}$, $\Omega_{M}=0.27$, and $\Omega_{\Lambda}=0.73$, so that 1 mas corresponds to a projected linear distance of $7.9 \mathrm{pc}$

$24 \mathrm{http}: / /$ web.bu.edu/blazars/VLBAproject.html

25 http://www.iaa.es/ iagudo/research/MAPCAT

$26 \mathrm{http}: / /$ james.as.arizona.edu/ psmith/Fermi
}

Lowell Observatory (1.83 m Perkins Telescope), San Pedro Mártir Observatory (0.84 m Telescope), Crimean Astrophysical Observatory ( $0.7 \mathrm{~m}$ Telescope), and St. Petersburg State University (0.4 m Telescope). Our total flux light curves (Figure 2) include data from the Fermi-Large Area Telescope (LAT) $\gamma$-ray $(0.1-200 \mathrm{GeV})$ and Swift-XRT X-ray $(2.4-10 \mathrm{keV})$ observatories, available from the archives of these missions, and $R X T E$ at $2.4-10 \mathrm{keV}$. Optical $R$-band fluxes come from the Tuorla Blazar Monitoring Program, ${ }^{27}$ the Yale University SMARTS program, ${ }^{28}$ and Maria Mitchell and Abastumani Observatories. Longer wavelength light curves were acquired from the Submillimeter Array (SMA) at $850 \mu \mathrm{m}$ and $1 \mathrm{~mm}$, the IRAM $30 \mathrm{~m}$ Telescope at $1 \mathrm{~mm}$, the Metsähovi $14 \mathrm{~m}$ Telescope at $8 \mathrm{~mm}$, and both the Owens Valley Radio Observatory (OVRO) $40 \mathrm{~m}$ Telescope Fermi Blazar Monitoring Program ${ }^{29}$ and University of Michigan Radio Astronomy Observatory ${ }^{30}$ (UMRAO) 26 m Telescope at $2 \mathrm{~cm}$.

We followed data reduction procedures described in previous studies-VLBA: Jorstad et al. (2005); optical polarimetric data: Jorstad et al. (2010); IRAM data: Agudo et al. (2006, 2010); SMA: Gurwell et al. (2007); Metsähovi: Teräsranta et al. (1998); OVRO: Richards et al. (2010); UMRAO: Aller et al. (1985); Swift: Jorstad et al. (2010); RXTE: Marscher et al. (2010); and Fermi-LAT: Marscher et al. (2010); Agudo et al. (2011). For both Swift and RXTE data, only the $2.4-10 \mathrm{keV}$ band is included in the X-ray light curve. In this energy band, where the intrinsic spectral curvature and photoelectric absorption at $z=0.524$ discussed by Raiteri et al. (2006, and references therein) are not important, a single absorbed power law (with Galactic absorption corresponding to a neutral hydrogen column density of $0.9 \times 10^{21} \mathrm{~cm}^{2}$ ) fit the data adequately. To process the $0.1-200 \mathrm{GeV}$ Fermi-LAT data, we used 2 day binning and a single power-law spectral fit with photon index held fixed at 2.14 (Abdo et al. 2010b). This produces essentially the same light curve as the broken-power-law spectral model of Abdo et al. (2010c).

\section{ANALYSIS OF OBSERVATIONAL RESULTS}

\subsection{Major Millimeter Flare in 2008 Related to a New Superluminal Knot}

Our 7 mm VLBA maps of $0235+164$ (Figure 1) reveal compact total intensity structure that is well fitted by one or two circular Gaussian components at most of the observing epochs, where the core generally corresponds to the brighter one. Between mid-2008 and mid-2009, the images also contain a second emission region (Qs, the brightest jet feature detected thus far in this object) that propagates at a superluminal apparent speed $\left\langle\beta_{\mathrm{app}}\right\rangle=(12.6 \pm 1.2) c$. The position angle of Qs $\in\left[\sim 150^{\circ}, \sim 180^{\circ}\right]$ contrasts with previously reported VLBI position angles by up to $\sim 180^{\circ}$, which is consistent with a sudden change of the jet ejection angle amplified by projection effects.

If the motion of Qs is ballistic, it was coincident with the core in $2008.30 \pm 0.08$, near the start of an extreme millimeterwave outburst (labeled $08_{\mathrm{mm}}$ in Figure 2) that peaked on 2008 October 10 with a flux density $\sim 6.5 \mathrm{Jy}$ at $3 \mathrm{~mm}$. Figure 2 shows that radio and millimeter-wave outbursts in $2008\left(08_{\text {rad }}\right.$ and $08_{\mathrm{mm}}$ ) contain contributions from both the core and

\footnotetext{
27 http://users.utu.fi/kani/1m

$28 \mathrm{http} / / / \mathrm{www}$.astro.yale.edu/smarts/glast

29 http://www.astro.caltech.edu/ovroblazars/

30 http://www.astro.lsa.umich.edu/obs/radiotel/
} 


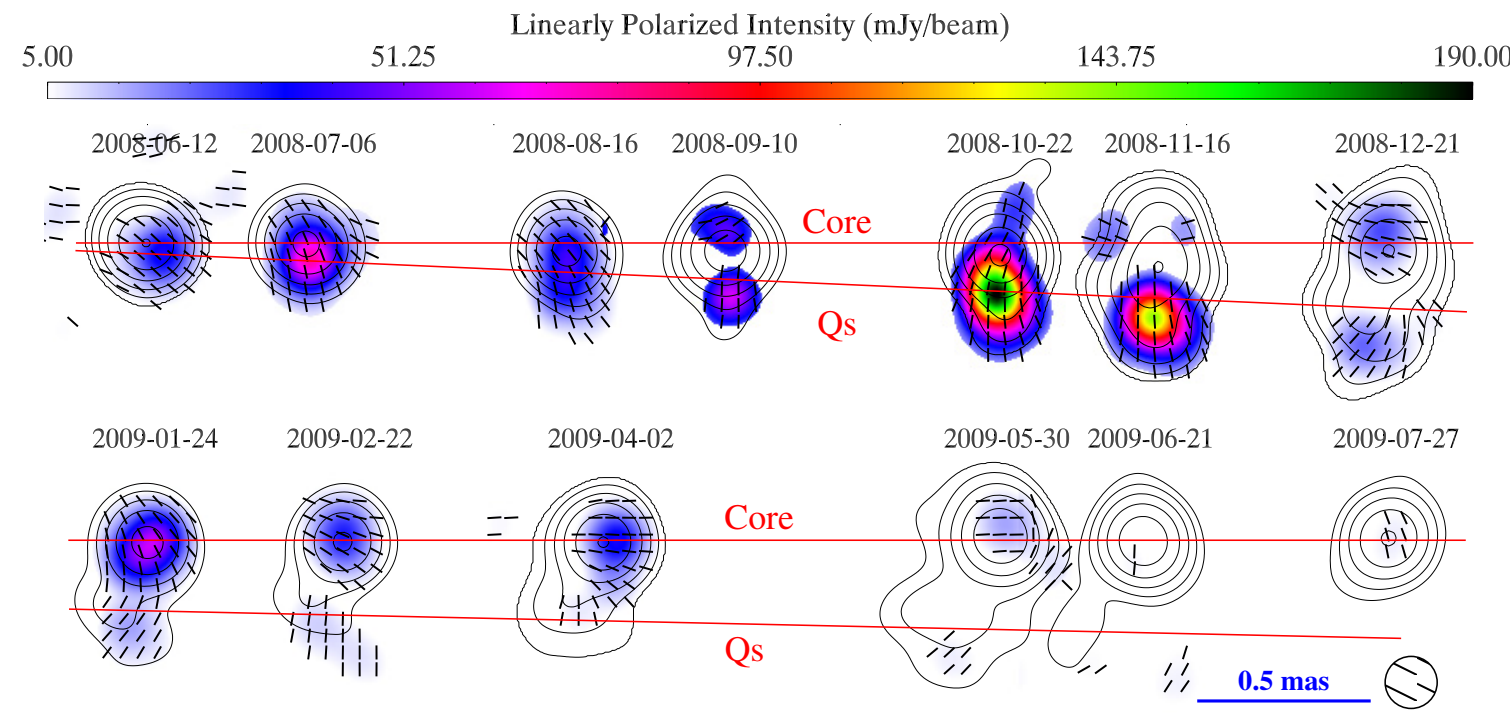

Figure 1. Sequence of $7 \mathrm{~mm}$ VLBA images of $0235+164$ convolved with an FWHM $=0.15$ mas circular Gaussian beam. Images in our program before 2008 June 12 and after 2009 July 27, containing only a single emission feature (i.e., the core), are not displayed. Contour levels represent total intensity (levels in factors of two from $0.4 \%$ to $51.2 \%$ plus $90.0 \%$ of peak $=4.93 \mathrm{Jy} \mathrm{beam}^{-1}$ ), color scale indicates polarized intensity, and superimposed sticks show the orientation of $\chi$.
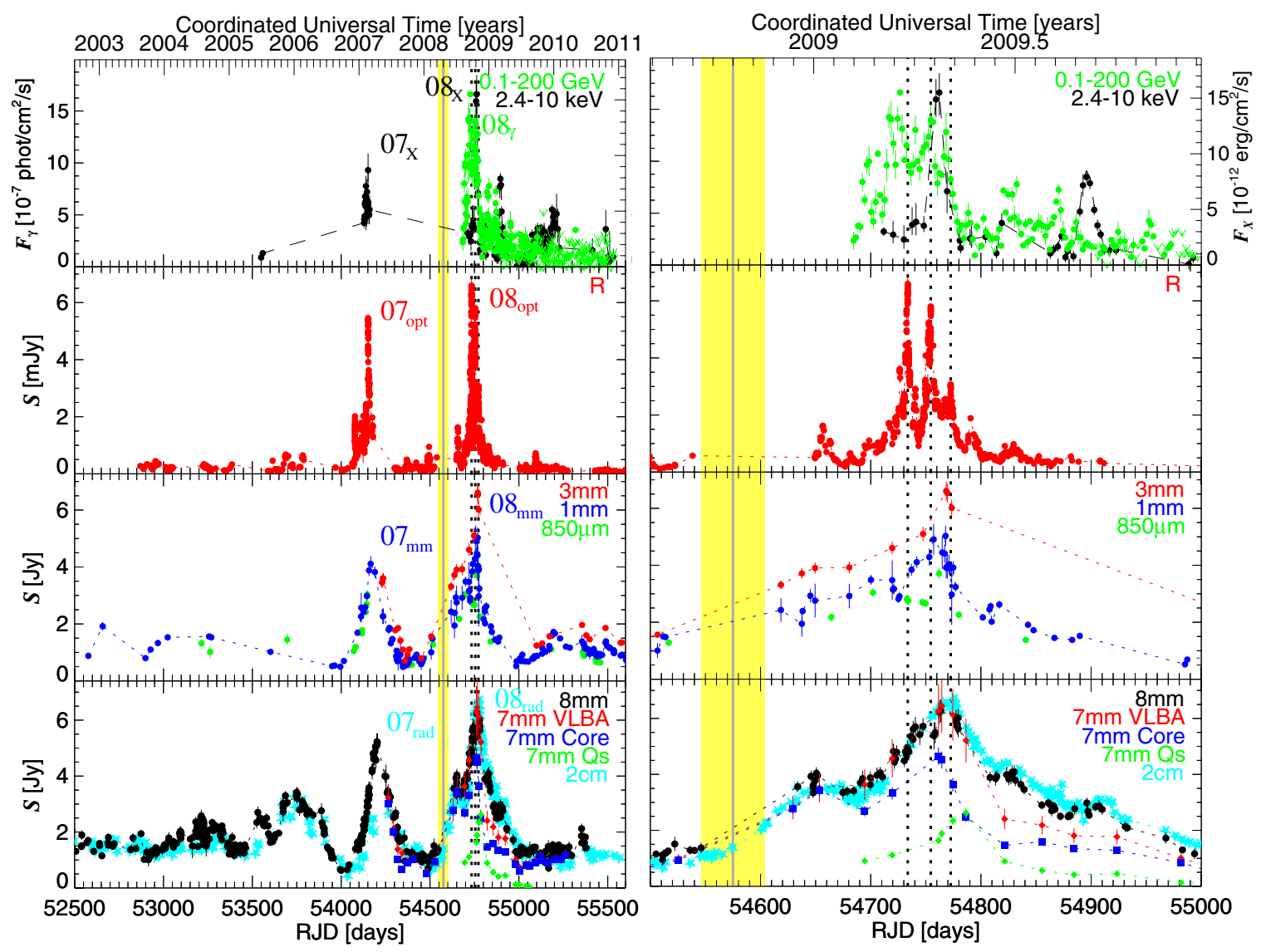

Figure 2. Left: light curves of $0235+164$ from $\gamma$-ray to millimeter wavelengths. Vertical dotted lines mark the three most prominent $08_{\text {opt }}$ optical peaks. The yellow area represents the time of ejection of feature Qs within its uncertainty. RJD = Julian Date -2400000.0 . Right: same as left panel for RJD $\in$ [54500, 55000].

Qs, whose fluxes reached maximum on 2008 October 20 and November 16, respectively. Their contemporaneous coevolution suggests that the disturbance responsible for the ejection of Qs extended from the location of the core to Qs in the frame of the observer, which could have resulted from lighttravel delays (e.g., Gómez et al. 1997; Agudo et al. 2001). Qs is the brighter $7 \mathrm{~mm}$ superluminal knot ever seen in $0235+164$, and flares $08_{\mathrm{rad}}$ and $08_{\mathrm{mm}}$ are the only outbursts that occurred after the ejection of Qs. The rarity of such events strongly implies that they are physically related.

The jet half-opening-angle of $0235+164\left(\alpha_{\text {int }} / 2 \lesssim 1.25\right)$ and the average FWHM of the core measured from our 31 VLBA observing epochs in $[2007,2010]\left(\left\langle\mathrm{FWHM}_{\text {core }}\right\rangle=\right.$ $(0.054 \pm 0.018)$ mas) constrain the $7 \mathrm{~mm}$ core to be at 

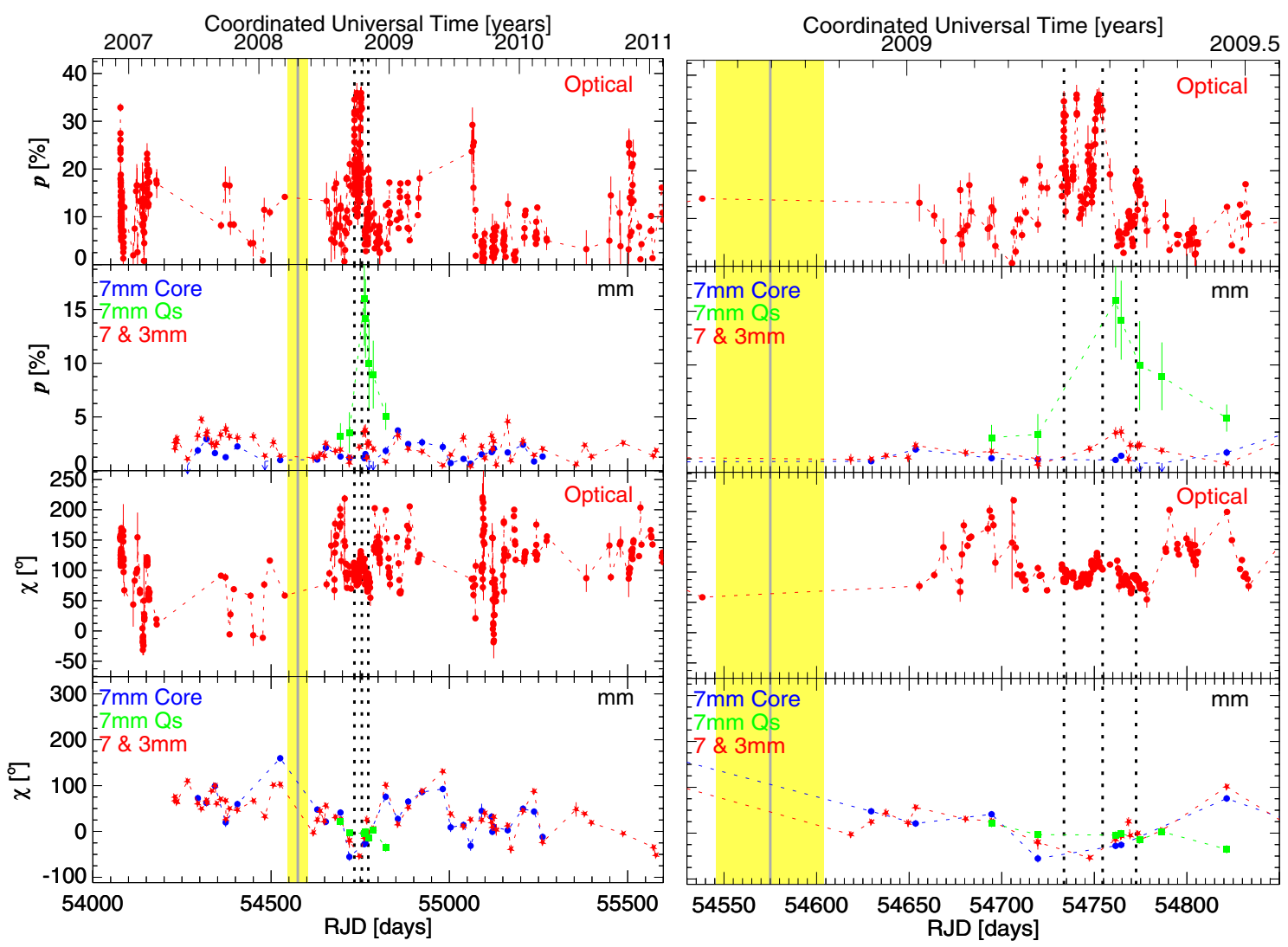

Figure 3. Left: long-term optical and millimeter-wave linear polarization evolution of $0235+164$ in the RJD $=[54000,55600]$ range. Right: same as left panel for $\mathrm{RJD} \in[54530,54850]$.

$d_{\text {core }}=1.8\left\langle\mathrm{FWHM}_{\text {core }}\right\rangle / \tan \alpha_{\mathrm{int}} \gtrsim 12 \mathrm{pc}$ from the vertex of the jet cone.

\subsection{Contemporaneous Flares from $\gamma$-ray to Radio Wavelengths}

Figure 2 reveals that the $08_{\text {rad }}$ and $08_{\mathrm{mm}}$ flares were accompanied by sharp optical, X-ray, and $\gamma$-ray counterparts $\left(08_{\text {opt }}\right.$, $08_{\mathrm{X}}$, and $08_{\gamma}$ flares, respectively). Our formal light curve correlation analysis (Figure 4)-performed following Agudo et al. (2011) - confirms the association of $\gamma$-ray variability with that at $2 \mathrm{~cm}, 8 \mathrm{~mm}, 1 \mathrm{~mm}$, and optical wavelengths at $>99.7 \%$ confidence. The flux evolution of the VLBI core is also correlated with the $\gamma$-ray light curve at $>99.7 \%$ confidence. Moreover, the evolution of the degree of optical linear polarization $\left(p_{\mathrm{opt}}\right)$ and $\mathrm{X}$-ray light curve are also correlated with the optical $R$ band, $1 \mathrm{~mm}$, and $2 \mathrm{~cm}$ light curves at $>99.7 \%$ confidence (Figure 5), further indicating that the extreme flaring activity revealed by our light curves is physically related at all wavebands from radio to $\gamma$-rays.

There is, however, no common pattern to the discrete correlation function (DCF) at all spectral ranges. This implies that, although there is correlation on long timescales (years), on short timescales $(\lesssim 2$ months) the variability pattern does not correspond as closely. This is the result of the intrinsic variability pattern rather than the irregular time sampling at some spectral ranges.

The sharp systematic peaks in the DCFs involving the $R$ band light curve aids in the identification of relative time delays across wavebands, as measured with regard to the first sharp $\mathrm{DCF}_{\mathrm{R}, \lambda 2}$ peak. In this way, we find that, relative to the $R$-band maximum, the peaks of the $\lambda 2=2 \mathrm{~cm}, 8 \mathrm{~mm}$, $1 \mathrm{~mm}$, and $7 \mathrm{~mm}$ core flares in 2008 are delayed by $\sim 60$, $\sim 45, \sim 40$, and $\sim 40$ days, respectively, whereas the X-ray delay is $\sim 25$ days. Only the $\gamma$-ray variations lead those at $R$ band, by $\sim 10$ days according to the peak in the DCF seen in Figure 4.

\subsection{Correlated Variability of Linear Polarization}

Figure 3 reveals extremely high, variable optical polarization, $p_{\text {opt }} \gtrsim 30 \%$, during the sharp $08_{\text {opt }}$ optical peaks (see also Hagen-Thorn et al. 2008, for the 2006-2007 outburst). Whereas the integrated millimeter-wave degree of linear polarization $\left(p_{\mathrm{mm}}\right)$ and that of the $7 \mathrm{~mm}$ core remain at moderate levels, $\lesssim 5 \%$, the polarization of Qs ( $\left.p_{\mathrm{mm}, \mathrm{Qs}}\right)$ peaks at the high value of $\sim 16 \%$ close to the time of the second sharp optical subflare. The coincidence of this sharp maximum of $p_{\mathrm{mm}, \mathrm{Qs}}$ in the brightest superluminal feature ever detected in $0235+164$ with the (1) high optical flux and polarization, (2) flares across the other spectral regimes, and (3) flare in the $7 \mathrm{~mm}$ VLBI core implies that the ejection and propagation of Qs in $0235+164$ 's jet is physically tied to the total flux and polarization variations from radio to $\gamma$-rays.

On long timescales (years), the linear polarization angle at both optical $\left(\chi_{\text {opt }}\right)$ and millimeter $\left(\chi_{\mathrm{mm}}\right.$ and $\left.\chi_{\mathrm{mm}}^{\text {core }}\right)$ wavelengths varies widely, without a preferred orientation or systematic common trend. However, during flare $08_{\text {opt }}, \chi_{\text {opt }}$ maintains a stable orientation at $(100 \pm 20)^{\circ}$, whereas $\chi_{\mathrm{mm}}^{\mathrm{Qs}}$ is roughly perpendicular to this $\left(\sim 0^{\circ}\right)$, as expected for a plane-perpendicular shock wave propagating to the south toward Qs. Owing to the large peak value of $\mathrm{Qs}, p_{\mathrm{mm}, \mathrm{Qs}}^{\max } \sim 16 \%$, one cannot explain the 

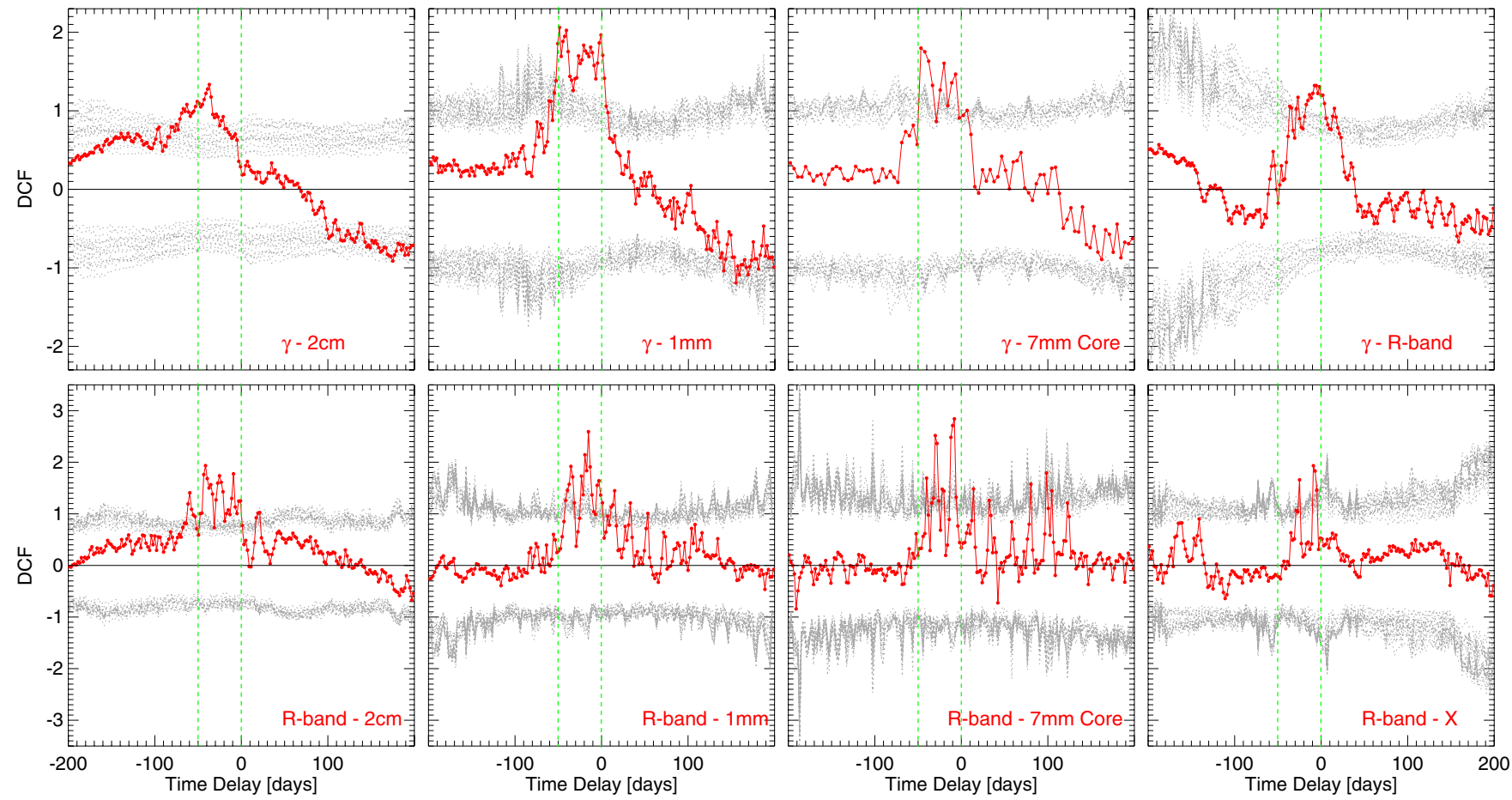

Figure 4. Grid of DCF (Edelson \& Krolik 1988) of labeled light curve pairs during the maximum time period RJD = [52200, 55600]. The top row of panels show DCF with $\gamma$-ray light curve, whereas the bottom row shows DCF with $R$-band light curve. Gray dotted curves at positive (negative) DCF values symbolize $99.7 \%$ confidence limits for correlation against the null hypothesis of stochastic variability. Each of these curves corresponds to a Monte Carlo simulation of $5000 \gamma$-ray and $\lambda 2$ (where $\lambda 2$ denotes the lower energy spectral range) light curves characterized by the same mean and standard deviation as those of the observed light curves, and by power-law power spectral densities (PSD $\propto 1 / f^{a}$ ) with $a_{\gamma} \in\{1.0,1.5,2.0\}$ and $a_{\lambda 2} \in\{1.0,1.5,2.0,2.5,3.0\}$. Green dashed lines at 0 and -50 day time lags are drawn for reference.
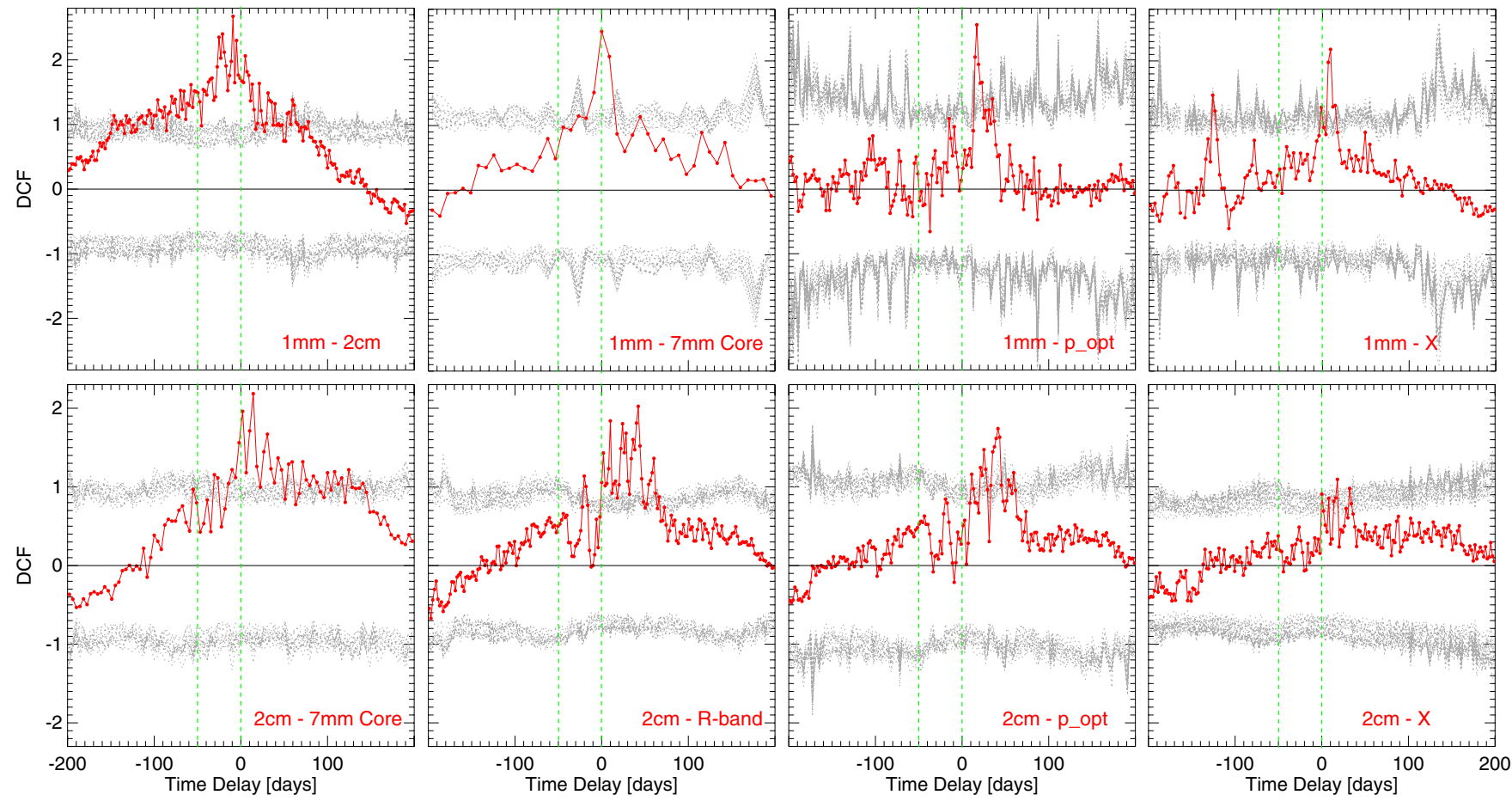

Figure 5. Same as Figure 4 but for the $1 \mathrm{~mm}$ light curve (top) and the $2 \mathrm{~cm}$ light curve (bottom).

orthogonal optical-millimeter polarizations by opacity effects. Instead, we propose that the optical polarization mainly arises in a conical shock associated with the $7 \mathrm{~mm}$ core, while the millimeter-wave polarization results from a propagating shock front associated with Qs. We surmise that the moving shock also emits polarized optical radiation, since the optical polarization drops precipitously when the orthogonal polarization of Qs peaks (Figure 3, right). 


\subsection{Low Probability of Chance Coincidences}

The relationship among the $\gamma$-ray, optical, and radiomillimeter flares is supported by probability arguments. If the flares occur randomly, the probability of observing, at any time, a $\gamma$-ray outburst like the one reported here (i.e., with flux $\gtrsim 10^{-6}$ photons $\mathrm{cm}^{-2} \mathrm{~s}^{-1}$ and duration $\sim 70$ days $)$ is $p_{\gamma}=0.08$. For optical and radio-millimeter wavelengths, this probability is $p_{\mathrm{opt}}=0.04$ and $p_{\mathrm{mm}}=0.15$, respectively. Thus, if the flares at different wavelengths were random and independent of each other, the probability of observing a $\gamma$-ray, optical, and radiomillimeter flare at any given time is $p_{\gamma, \mathrm{opt}, \mathrm{mm}}=5 \times 10^{-4}$. This counters the null hypothesis of random coincidence at $99.95 \%$ confidence. This confidence keeps $>99.6 \%$ even when unrealistically considering twice as long as observed $\gamma$-ray, optical, and radio-millimeter flares, thus pointing out the robustness of this result.

\section{DISCUSSION AND CONCLUSIONS}

The coincidence of the ejection and propagation of Qs - by far the brightest non-core feature ever reported in $0235+164$ - with the prominent $\gamma$-ray to radio outbursts and the extremely high values of $p_{\mathrm{opt}}$ and $p_{\mathrm{mm} \text {, Qs }}$ provides convincing evidence that all these events are physically connected. This is supported by probability arguments and by our formal DCF analysis, which unambiguously confirms the relation of the $\gamma$-ray outburst in late 2008 with those in the optical, millimeter-wave (including the $7 \mathrm{~mm}$ VLBI core), and radio regimes. Comprehensive studies of other blazars place the core parsecs downstream of the $\mathrm{BH}$ (Jorstad et al. 2010; Marscher et al. 2008, 2010; Agudo et al. 2011).

The general correlation of $\gamma$-ray and optical light curves (Figures 4 and 5) contrasts with the poor detailed correspondence during the main outburst (Figure 2). This is difficult to reconcile with the external Compton mechanism, in which $\gamma$-rays arise from inverse Compton scattering of photons originating outside the jet by electrons in the jet (Begelman \& Sikora 1987; Dermer \& Schlickeiser 1993). According to this model, rapid fluctuations of the $\gamma$-ray and optical flux are caused by changes in the number of electrons with sufficient energy to radiate at these frequencies. However, such changes should affect the flux at both wavebands in a similar manner if the seed photon field varies smoothly with distance down the jet, as expected if these photons are from the broad emission-line region (Sikora et al. 1994) or a dust torus (Błażejowski et al. 2000; Malmrose et al. 2011). This is contrary to our observations unless the magnetic field varies in a much different manner than does the energy density of relativistic electrons. Instead, the nature of the variations suggests that the seed photon field also varies quite rapidly.

A naturally variable seed photon source is synchrotron radiation from the jet. The maximum apparent $\gamma$-ray luminosity of $0235+164$ is similar to that of the optical synchrotron radiation (Abdo et al. 2010a). Thus, synchrotron self-Compton (SSC) radiation can potentially explain the $\gamma$-ray emission without leading to higher-order scattering that flattens the $\gamma$-ray spectrum. We can reconcile the short timescales of variability, $\lesssim 1$ day, with a distance of parsecs from the $\mathrm{BH}$, by appealing to both the very narrow opening angle of the jet (see above) and the possibility of structure across the jet. The former limits the width of the emission region to $\sim 0.8 \mathrm{pc}$, while the latter allows individual zones of emission to be no larger than $\sim 0.8 / \sqrt{N}$ pc, where $N$ is the number of zones, which presum- ably represent turbulent cells. In fact, Marscher \& Jorstad (2010) used $0235+164$ as a blazar whose spectral energy distribution, polarization, and variability properties can be well represented by such a model. The synchrotron flux varies in response to changes in the energy density of the flow, maximum electron energy, and magnetic field direction in each cell, while the SSC flux varies from the first two of these plus the time-delayed synchrotron photon density from the other cells. These variations follow similar trends, leading to a general correlation but with detailed fluctuations that can differ significantly.

We identify the $7 \mathrm{~mm}$ core as the first re-collimation shock near the end of the jet's acceleration and collimation zone (Jorstad et al. 2007; Marscher et al. 2008, 2010). Superluminal feature Qs is consistent with a moving shock oriented transverse to the jet axis, given the extremely high $p_{\mathrm{mm}, \mathrm{Qs}}$, with $\chi_{\mathrm{mm}}^{\mathrm{Qs}}$ parallel to the direction of propagation of Qs. The flux evolution of the core appears closely tied to that of Qs, and its light curve is correlated at high confidence with those at $\gamma$-ray, optical, and millimeter wavelengths. This suggests that Qs is the head of an extended disturbance, perhaps containing a front-back structure stretched by light-travel delays in the observer's frame (see, e.g., Aloy et al. 2003). The presence of this dual structure is also consistent with the perpendicular $\chi_{\mathrm{opt}}$ and $\chi_{\mathrm{mm}}^{\mathrm{Qs}}$ if Qs corresponds to the front region emitting at millimeter wavelengths, while the optical flare arises mainly from the interaction of the slower back region with the standing conical shock in the core.

Under this scenario, the radio/millimeter-wave and optical (and perhaps X-ray) synchrotron flares start when the front region crosses the conical shock at the core, where the jet is at least partially optically thin. This interaction accelerates electrons, increasing the synchrotron emissivity, and produces SSC $\gamma$-ray emission from the upscattering of IR-optical photons. There is a time delay of the latter, since the seed photons must travel across part of the jet before arriving at the scattering site (see Sokolov et al. 2004). When the back region of the moving perturbation encounters the core, their interaction again produces efficient particle acceleration, which is seen as a sudden optical and radio/millimeter synchrotron emission enhancement. The subsequent optical variability is produced by the passage of the remaining shocked turbulent plasma in the back structure through the core. During the different optical sub-flares, the integrated radio/millimeter synchrotron flux keeps rising. This radio/millimeter outburst is more prolonged owing to the longer synchrotron cooling time of electrons radiating at these wavelengths and the lower speed of the back structure. Indeed, Qs does not reach its maximum radio/millimeter-wave flux until traveling a projected distance of $\sim 0.13$ mas from the core.

When the entire front-back structure passes across the core, the synchrotron emission declines rapidly at optical (and, if relevant, X-ray) frequencies, as does the $\gamma$-ray SSC emission. The decay of the radio/millimeter-wave emission is more gradual (see above). The prominent X-ray flare on RJD 54760-54770 is consistent with being simultaneous with the peak of a millimeter and/or optical synchrotron flare and could arise from either scattering of millimeter photons or synchrotron radiation from the highest-energy electrons. The former is consistent with the evolution of the X-ray spectral index, which gradually steepens to values consistent with the optically thin millimeter-IR spectral index $(\sim 1)$ at the time of the $X$-ray peak.

We acknowledge the anonymous referee for constructive comments. This research was funded by NASA grants NNX08AJ64G, NNX08AU02G, NNX08AV61G, and 
NNX08AV65G, NSF grant AST-0907893, and NRAO award GSSP07-0009 (Boston University); RFBR grant 09-02-00092 (St. Petersburg State University); MICIIN grant AYA201014844, and CEIC (Andalucía) grant P09-FQM-4784 (IAACSIC); the Academy of Finland (Metsähovi); NASA grants NNX08AW56S and NNX09AU10G (Steward Observatory); and GNSF grant ST08/4-404 (Abastunami Observatory). The VLBA is an instrument of the NRAO, a facility of the NSF under cooperative agreement by AUI. The PRISM camera was developed by Janes et al. and funded by NSF, Boston University, and Lowell Observatory. Calar Alto Observatory is operated by MPIA and IAA-CSIC. The IRAM $30 \mathrm{~m}$ Telescope is supported by INSU/CNRS, MPG, and IGN. The SMA is a joint project between the SAO and the Academia Sinica.

\section{REFERENCES}

Abdo, A. A., et al. 2010a, ApJ, 716, 30

Abdo, A. A., et al. 2010b, ApJS, 188, 405

Abdo, A. A., et al. 2010c, ApJ, 710, 1271

Ackermann, M., et al. 2010, ApJ, 721, 1383

Agudo, I., Gómez, J.-L., Martí, J.-M., Ibáñez, J.-M., Marscher, A. P., Alberdi, A., Aloy, M.-A., \& Hardee, P. E. 2001, ApJ, 549, L183

Agudo, I., Thum, C., Wiesemeyer, H., \& Krichbaum, T. P. 2010, ApJS, 189,

Agudo, I., et al. 2006, A\&A, 456, 117

Agudo, I., et al. 2011, ApJ, 726, L13

Aller, H. D., Aller, M. F., Latimer, G. E., \& Hodge, P. E. 1985, ApJS, 59, 513

Aloy, M.-Á., Martí, J.-M., Gómez, J.-L., Agudo, I., Müller, E., \& Ibáñez, J.-M. 2003, ApJ, 585, L109

Balonek, T. J., \& Dent, W. A. 1980, ApJ, 240, L3

Begelman, M. C., \& Sikora, M. 1987, ApJ, 322, 650

Błażejowski, M., Sikora, M., Moderski, R., \& Madejski, G. M. 2000, ApJ, 545, 107

Dermer, C. D., \& Schlickeiser, R. 1993, ApJ, 416, 458

Edelson, R. A., \& Krolik, J. H. 1988, ApJ, 333, 646
Foschini, L., Ghisellini, G., Tavecchio, F., Bonnoli, G., \& Stamerra, A. 2011, A\&A, 530, A77

Foschini, L., Tagliaferri, G., Ghisellini, G., Ghirlanda, G., Tavecchio, F., \& Bonnoli, G. 2010, MNRAS, 408, 448

Gómez, J. L., Martí, J.-M., Marscher, A. P., Ibáñez, J.-M., \& Alberdi, A. 1997, ApJ, 482, L33

Gopal-Krishna, Wiita, P. J., \& Dhurde, S. 2006, MNRAS, 369, 1287

Gurwell, M. A., Peck, A. B., Hostler, S. R., Darrah, M. R., \& Katz, C. A. 2007, in ASP Conf. Ser. 375, From Z-Machines to ALMA: (Sub)millimeter Spectroscopy of Galaxies, ed. A. J. Baker et al. (San Francisco, CA: ASP), 234

Hagen-Thorn, V. A., Larionov, V. M., Jorstad, S. G., Arkharov, A. A., HagenThorn, E. I., Efimova, N. V., Larionova, L. V., \& Marscher, A. P. 2008, ApJ, 672,40

Hovatta, T., Valtaoja, E., Tornikoski, M., \& Lähteenmäki, A. 2009, A\&A, 494, 527

Jorstad, S. G., Marscher, A. P., Mattox, J. R., Wehrle, A. E., Bloom, S. D., \& Yurchenko, A. V. 2001, ApJS, 134, 181

Jorstad, S. G., et al. 2005, AJ, 130, 1418

Jorstad, S. G., et al. 2007, AJ, 134, 799

Jorstad, S. G., et al. 2010, ApJ, 715, 362

Ledden, J. E., Aller, H. D., \& Dent, W. A. 1976, Nature, 260, 752

Malmrose, M., Marscher, A., Jorstad, S., Nikutta, R., \& Elitzur, M. 2011, ApJ, 732,116

Marscher, A. P., \& Jorstad, S. G. 2010, in Fermi Meets Jansky-AGN at Radio and Gamma-rays, ed. T. Savolainen et al. (Bonn: Max-Planck-Institute Für Radioastronomie), 171

Marscher, A. P., et al. 2008, Nature, 452, 966

Marscher, A. P., et al. 2010, ApJ, 710, L126

Piner, B. G., Bhattarai, D., Edwards, P. G., \& Jones, D. L. 2006, ApJ, 640, 196

Raiteri, C. M., Villata, M., Kadler, M., Krichbaum, T. P., Böttcher, M., Fuhrmann, L., \& Orio, M. 2006, A\&A, 452, 845

Raiteri, C. M., et al. 2008, A\&A, 480, 339

Raiteri, C. M., et al. 2009, A\&A, 507, 769

Richards, J. L., et al. 2010, ApJS, submitted (arXiv:1011.3111)

Sikora, M., Begelman, M. C., \& Rees, M. J. 1994, ApJ, 421, 153

Sokolov, A., Marscher, A. P., \& McHardy, I. M. 2004, ApJ, 613, 725

Tavecchio, F., Ghisellini, G., Bonnoli, G., \& Ghirlanda, G. 2010, MNRAS, 405, L94

Teräsranta, H., et al. 1998, A\&AS, 132, 305 